\title{
Design and production of a novel antimicrobial fusion protein in Escherichia coli
}

Baode Sun, ${ }^{1}$ David Wibowo, ${ }^{1,2^{*}}$ Frank Sainsbury, ${ }^{1}$ Chun-Xia Zhao ${ }^{1 *}$

${ }^{1}$ Australian Institute for Bioengineering and Nanotechnology, The University of Queensland, St Lucia QLD 4072, Australia.

${ }^{2}$ Griffith Institute for Drug Discovery, Griffith University, Nathan QLD 4111, Australia.

\section{*Correspondence}

David Wibowo. Email: d.wibowo@griffith.edu.au. ORCID ID: 0000-0001-6919-3355

Chun-Xia Zhao. Email: z.chunxia@uq.edu.au. ORCID ID: 0000-0002-3365-3759

\section{Keywords}

antimicrobial agents; fusion proteins; minimum inhibitory concentration; protein purification; recombinant $E$. coli

\begin{abstract}
In recent years, antimicrobial peptides (AMPs) have attracted increasing attention. The microbial cells provide a simple, cost-effective platform to produce AMPs in industrial quantities. While AMP production as fusion proteins in microorganisms is commonly used, the recovery of AMPs necessitates the use of expensive proteases and extra purification steps. Here we develop a novel fusion protein DAMP4-F-pexiganan comprising a carrier protein DAMP4 linked to the AMP, pexiganan, through a long, flexible linker. We show that this fusion protein can be purified using a non-chromatography approach and exhibits the same antimicrobial activity as the chemically synthesized pexiganan peptide without any cleavage step. Activity of the fusion protein is dependent on a long, flexible linker between the AMP and carrier domains, as well as on the expression conditions of the fusion protein, with low-temperature expression promoting better folding of the AMP domain. The production of DAMP4-F-pexiganan circumvents the time-consuming and costly steps of chromatography-based purification and enzymatic cleavages, therefore shows considerable advantages over traditional microbial production of AMPs. We expect this novel fusion protein, and the studies on the effect of linker and expression conditions on its antimicrobial activity, will broaden the rational design and production of antimicrobial products based on AMPs.
\end{abstract}




\section{Introduction}

Antibiotic resistance has become a serious global health issue (Davies and Davies 2010). There is an urgent demand for the development of new drug or treatment strategies to overcome drug resistance. Antimicrobial peptides (AMPs), as a promising alternative to small molecule antimicrobials for the treatment of antibioticresistant pathogens, have attracted increasing attention during the past decade (Ageitos et al. 2017; Fox 2013). AMPs are a group of naturally occurring small and highly charged peptides, that make up an essential part of the innate immune system of varied organisms (Guaní-Guerra et al. 2010; Pasupuleti et al. 2012). Not only do AMPs display broad spectrum antimicrobial activities against pathogens, but microorganisms are also less prone to develop resistance to AMPs since their antimicrobial activities result from a combination of different mechanisms (Brogden 2005). Compared to conventional production strategies of AMPs (e.g., isolation from natural sources or chemical synthesis), microbial production systems are regarded as an attractive platform for producing AMPs efficiently and cost-effectively (Parachin et al. 2012). Escherichia coli is the most frequently used microbial platform to produce AMPs due to well-characterized methods for genetic engineering, rapid growth and high recombinant protein yields ( $\mathrm{Li}$ 2011).

To avoid the potential toxicity of AMPs toward the host cells and reduce the susceptibility of AMPs to proteolysis during production and purification, AMPs produced in microbial systems are frequently conjugated to a carrier protein (Li 2011; Parachin et al. 2012). Two types of carrier proteins are frequently used for the production of AMPs: solubility-enhancing carriers and aggregation-promoting carriers (Li 2009). Solubility-enhancing carriers such as small ubiquitin-related modifiers and thioredoxin can facilitate production of soluble fusion proteins and reduce the toxicity of AMPs toward host cells by neutralizing the net charge of AMPs (Li et al. 2011; Zhou et al. 2007). By contrast, aggregation-promoting carriers (e.g., PurF fragment and ketosteroid isomerase) lead to the production of insoluble fusion proteins, masking toxicity of AMPs (Lee et al. 2000; Majerle et al. 2000). However, collection and purification of the fusion protein from cell lysates remain labor intensive and costly (Parachin et al. 2012). Isolation of the fusion proteins having solubility-enhancing carriers commonly requires expensive affinity-based chromatographic processes (Capparelli et al. 2007). Although insoluble fusion proteins with aggregation-promoting carriers can be collected efficiently by centrifugation, refolding and re-solubilizing steps are required to recover active fusion proteins (Lee et al. 2000; Vallejo and Rinas 2004).

In addition, to obtain active AMPs, the subsequent release of AMPs from the fusion proteins is needed. Hence, linkers used in the AMP-fused proteins commonly contain recognition sites, which allow the release of active AMPs by chemical (e.g., CNBr and formic acid) or enzymatic cleavage (e.g., thrombin and TEV protease) (Li 2009; Parachin et al. 2012). Enzymatic cleavage relies on expensive enzymes and carefully controlled reaction conditions. Moreover, it is dependent on the structure of the fusion protein, which may hinder the accessibility of the recognition site to enzymes (Zhou et al. 2007). Chemical cleavage can be more efficient and less expensive (Gibbs et al. 2004). However, it suffers from several disadvantages, including residual toxic chemicals, and typically harsh conditions that result in side chain modification of AMPs (Toennies and Homiller 1942).

In this work, we designed a new recombinant protein by fusing an antimicrobial peptide, pexiganan (GIGKFLKKAKKFGKAFVKILKKHH), to a four-helix bundle protein, DAMP4 (MD(PSMKQLADSLHQLARQ-VSRLEHAD) 4 ) using a long, flexible linker (PGGGGSGGGGSLVPRGS), called DAMP4-Fpexiganan (Middelberg and Dimitrijev-Dwyer 2011). The DAMP4-F-pexiganan protein is able to be produced in E. coli cells and purified through a simple, non-chromatographic method (Dwyer et al. 2014). We assessed the 
effect of different linkers on the antimicrobial activity of the fusion protein and investigated the impact of different expression conditions on purification, yield and antimicrobial performance of DAMP4-F-pexiganan. The results illustrate that the long, flexible linker plays a critical role in retaining the antimicrobial activity of the AMP and suggest that low-temperature expression facilitates the correct folding of AMP domain of the fusion proteins.

\section{Materials and methods}

\section{Materials}

Pexiganan peptide (GIGKFLKKAKKFGKAFVKILKK-NH 2 , molecular weight (MW) 2477.17 Da) was custom synthesized by Genscript Corporation (Piscataway, NJ) with purity $>99 \%$. All the chemicals were of analytical grade from Sigma-Aldrich (St Louis, MO) unless otherwise noted. A stock solution of poly(ethyleneimine) (PEI) $5 \%(\mathrm{w} / \mathrm{v})$ at $\mathrm{pH} 8$ was prepared by adding hydrochloric acid. Ultrapure water (with $>18.2 \mathrm{M} \Omega \cdot \mathrm{cm}$ resistivity) was produced by a Milli-Q system with a $0.22 \mu \mathrm{m}$ filter (Millipore, North Ryde, Australia) and used in all the experiments.

\section{Construction of pET48b-DAMP4-F-pexiganan fusion plasmid}

The DNA sequence of DAMP4-F-pexiganan (GenBank accession number: MH532953) was codon optimized for E. coli and synthesized by Integrated DNA Technologies (Baulkham Hills, Australia) with 5' and 3' extensions corresponding to sequences upstream and downstream of the expression vector insertion site. Oligonucleotide primers corresponding to these extensions (Table S1) were used to amplify the DAMP4-F-pexiganan gene and linearize pET-48b(+) plasmid (Novagen, North Ryde, Australia) via polymerase chain reaction (PCR). Then the PCR product of DAMP4-F-pexiganan gene and linearized pET-48b(+) plasmid were assembled using NEBuilder ${ }^{\circledR}$ HiFi DNA Assembly Master Mix (Genesearch, Arundel, Australia) to construct the expression vector pET48bDAMP4-F-pexiganan. The resulting vector was amplified by transforming the vector into $\mathrm{NEB}^{\circledR}$ 5-alpha E. coli cells (Genesearch, Arundel, Australia) following its standard transformation protocol. The coding sequence of pET48b-DAMP4-F-pexiganan was confirmed by Sanger sequencing and then the vector was transformed into chemically competent $E$. coli BL21(DE3) (Novagen, Merck KGaA, Germany). The successfully transformed $E$. coli BL21(DE3) was preserved as glycerol stock and stored at $-80^{\circ} \mathrm{C}$.

\section{Production of DAMP4-F-pexiganan}

Glycerol-stocked cells of $E$. coli strain BL21(DE3) harboring pET48b-DAMP4-F-pexiganan plasmid was firstly streaked on Luria Bertani (LB) agar plates (15 g/L agar, $10 \mathrm{~g} / \mathrm{L}$ tryptone, 5 g/L yeast extract, $10 \mathrm{~g} / \mathrm{L} \mathrm{NaCl}$ ) and incubated at $37^{\circ} \mathrm{C}$ overnight. Then, a single colony was selected from the plates and inoculated into $5 \mathrm{~mL} \mathrm{LB}$ media (15 g/L agar, $10 \mathrm{~g} / \mathrm{L}$ tryptone, $5 \mathrm{~g} / \mathrm{L}$ yeast extract), and grown in a shaking incubator at $37^{\circ} \mathrm{C}, 180 \mathrm{rpm}$ overnight. The overnight culture ( $\left.400 \mu \mathrm{L}, \mathrm{OD}_{600} \sim 2\right)$ was further inoculated into $800 \mathrm{~mL} 2 \times$ yeast extract and tryptone media (16 g/L tryptone, $10 \mathrm{~g} / \mathrm{L}$ yeast extract, $5 \mathrm{~g} / \mathrm{L} \mathrm{NaCl})$, followed by an incubation at $37^{\circ} \mathrm{C}, 180 \mathrm{rpm}$ to an $\mathrm{OD}_{600}$ of 0.5 . E. coli was induced with $1 \mathrm{mM}$ isopropyl- $\beta$-D-thiogalactopyranoside (IPTG) (Astral Scientific, Taren Point) at $25^{\circ} \mathrm{C}, 16 \mathrm{~h}$. The cell pellets were collected by centrifugation $\left(4,000 \times \mathrm{g}, 4^{\circ} \mathrm{C}, 20 \mathrm{~min}\right)$ and stored at $-20^{\circ} \mathrm{C}$. All bacterial-cell media were supplemented with $50 \mu \mathrm{g} / \mathrm{mL}$ kanamycin sulfate.

\section{Non-chromatographic purification of DAMP4-F-pexiganan}

The protein purification followed a standard protocol for DAMP4-based protein (Sun et al. 2018). Briefly, the cell

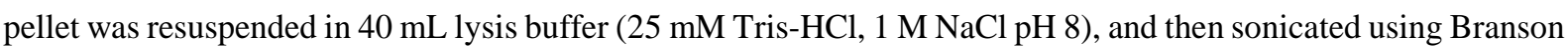
Sonifier 250 ultrasonicator (Branson Ultrasonics, Danbury, CT) at the power level of $60 \mathrm{~W}$ for four bursts of $30 \mathrm{~s}$ while keeping the cell extract cool. Supernatant was collected after centrifugation $\left(38,000 \times \mathrm{g}, 4^{\circ} \mathrm{C}, 20 \mathrm{~min}\right)$, 
followed by adding $5 \%(\mathrm{w} / \mathrm{v})$ PEI solution (pH 8$)$ to a final concentration of $0.5 \%(\mathrm{w} / \mathrm{v})$ with stirring under $4^{\circ} \mathrm{C}$ for $1 \mathrm{~h}$. After centrifugation $\left(38,000 \times \mathrm{g}, 4^{\circ} \mathrm{C}, 20 \mathrm{~min}\right), \mathrm{Na}_{2} \mathrm{SO}_{4}$ (solid) was added into the supernatant to a final concentration of $1 \mathrm{M}$, and the mixture was heated at $90^{\circ} \mathrm{C}$ for $30 \mathrm{~min}$ with stirring. Subsequently, the supernatant was separated and collected following centrifugation $\left(38,000 \times \mathrm{g}, 25^{\circ} \mathrm{C}, 20 \mathrm{~min}\right)$. More $\mathrm{Na}_{2} \mathrm{SO}_{4}$ (solid) was added to a final concentration of $1.8 \mathrm{M}$ and stirred at $35^{\circ} \mathrm{C}$ for $1 \mathrm{~h}$. The precipitate was collected by centrifugation (38,000×g, $4^{\circ} \mathrm{C}, 20 \mathrm{~min}$ ), and then washed with rinsing buffer ( $25 \mathrm{mM}$ Tris- $\mathrm{HCl}, 1 \mathrm{M} \mathrm{NaCl}, 2.4 \mathrm{M} \mathrm{Na}_{2} \mathrm{SO}_{4}, \mathrm{pH}$ 8). The final precipitate collected after centrifugation $\left(38,000 \times \mathrm{g}, 25^{\circ} \mathrm{C}, 20 \mathrm{~min}\right)$ was solubilized with the solubilizing buffer (25 mM Tris- $\mathrm{HCl}, 1 \mathrm{M} \mathrm{NaCl}, \mathrm{pH}$ 8), and then dialyzed against 4-(2-hydroxyethyl)-1piperazineethanesulfonic acid (HEPES) buffer $\left(10 \mathrm{mM}, \mathrm{pH}\right.$ 7.2) using SnakeSkin ${ }^{\circledR}$ dialysis membrane $(10,000$ molecular weight cut off) (Thermo Fisher Scientific, North Ryde, Australia).

\section{Analytical characterization}

Sodium dodecyl sulfate polyacrylamide gel electrophoresis (SDS-PAGE) was performed using NuPAGE 4-12\% Bis-Tris Precast Gels with an XCell SureLock ${ }^{\mathrm{TM}}$ Mini-Cell Electrophoresis System (Life Technologies, Mulgrave, Australia) and 2-(N-morpholino) ethanesulfonic acid buffer. Novex BenchMark Protein Ladder (Life Technologies) and Precision Plus Protein ${ }^{\mathrm{TM}}$ Dual Xtra Prestained Protein Standards (Bio-Rad, Hercules, CA) were used as the standards of protein molecular weight. Sample preparation, staining, and destaining were performed as recommended by the manufacturer.

Quantification of proteins was carried out using analytical reversed-phase high-performance liquid chromatography (RP-HPLC) on an LC-10AVP HPLC system (Shimadzu, Kyoto, Japan) equipped with a Jupiter $300 \AA$ Å C18 column (Phenomenex, Torrance, CA). Buffer A was 0.1\% trifluoroacetic acid (TFA) in water, and buffer B was $90 \%$ acetonitrile, $0.1 \%$ TFA in water. Gradient conditions were applied from 30 to $65 \%$ of buffer B at a flow rate of $1 \mathrm{~mL} / \mathrm{min}$ in $35 \mathrm{~min}$ and a detection wavelength of $214 \mathrm{~nm}$ was set.

Liquid chromatography-mass spectrometry was employed to identify the molecular weight of the protein and peptide samples using a Waters Alliance HPLC system (Waters, Milford, MA) coupled to a Quattro Micro API Quadrupole system (Waters, Milford, MA). The Liquid chromatography was equipped with a Kinetex C18 column (100 A, $2.6 \mu \mathrm{m}, 100 \mathrm{~mm} \times 4.6 \mathrm{~mm}$, Phenomenex, Torrance, CA) with mobile phases A $(0.01 \%$ TFA in water) and B (90\% acetonitrile, $0.01 \%$ TFA in water). An applied gradient from 30 to $65 \%$ in 35 min at column flow rate of $0.6 \mathrm{~mL} / \mathrm{min}$ was used. A detection wavelength was set at $214 \mathrm{~nm}$. For mass analysis, an electrospray mass spectrometry (MS) in positive-ion mode was performed with capillary, cone, extractor, and a radio-frequency lens voltage set at $3 \mathrm{kV}, 24 \mathrm{~V}, 3 \mathrm{~V}$, and $0 \mathrm{~V}$, respectively.

\section{Antimicrobial activity assay}

Minimum inhibitory concentration (MIC) tests of proteins and peptides were performed to evaluate their antimicrobial activities using broth microdilution method.(Wiegand et al. 2008) In brief, protein or peptide samples were introduced into a 96-well plate (Costar 3596, Corning, NY) with a serial dilution using Milli-Q water to a final volume of $90 \mu \mathrm{L}$ and a final concentration of $10 \mu \mathrm{M}$. E. coli ATCC $^{\circledR} 25922^{\mathrm{TM}}$ (Manassas, VA) was used as the test strain and grown in Mueller-Hinton (MH) broth (Becton Dickinson, Sparks, MD) at $37^{\circ} \mathrm{C}, 180 \mathrm{rpm}$ until an $\mathrm{OD}_{600}$ of 0.5 . The bacteria were pelleted by centrifugation $\left(4,000 \times \mathrm{g}, 4^{\circ} \mathrm{C}, 20 \mathrm{~min}\right)$ and resuspended in fresh $\mathrm{MH}$ broth to an $\mathrm{OD}_{600}$ of 0.35 . Subsequently, $10 \mu \mathrm{L}$ of the bacterial suspension was added into each well containing the protein or peptide samples, followed by incubation at room temperature for $16 \mathrm{~h}$. The MIC values of proteins and peptides were then determined by visual inspection. 


\section{Results}

In this paper, a cost-effective process was developed to produce DAMP4-F-pexiganan based on the microbial factories and a non-chromatography purification method. Besides that, we investigated the antimicrobial activity of the fusions with different linker lengths, as well as the effects of expression condition on the purification results and antimicrobial activity of the fusion protein.

\section{Design of DAMP4-F-pexiganan}

In this paper, DAMP4-F-pexiganan (MD(PSMKQLADSLHQLARQVSRLEHAD) ${ }_{4}$ PGGGGSGGGGSLVPRGS GIGKFLKKAKKFGKAFVKILKKHH) having a MW of 15188.78 was designed by fusing DAMP4 protein with a modified pexiganan peptide through a linker, which structure is schematically shown in Fig. 1. DAMP4 is a designed hyperstable four-helix bundle protein (Middelberg and Dimitrijev-Dwyer 2011). and was selected here as a protein carrier to facilitate both high-level fusion protein production and non-chromatographic purification (Sun et al. 2018; Wibowo et al. 2017; Zhao et al. 2015). DAMP4 has the capacity to maintain high solubility and structural stability under high temperature (up to $110^{\circ} \mathrm{C}$ ) and high salt concentrations under which most host protein precipitate (Dwyer et al. 2014). Moreover, the small size of DAMP4 protein (MW = 11,157.52 Da) enables the target AMP to constitute a large percentage of the purified fusion protein. Pexiganan (GIGKFLKKAKKFGKAFVKILKK) is a commercially-available antimicrobial peptide having a broad-spectrum activity against pathogenic bacteria (Gottler and Ramamoorthy 2009), and its modified sequence pexiganan-HH (GIGKFLKKAKKFGKAFVKILKKHH) was used based on our previous studies (Zhao et al. 2015). The linker connecting the protein carrier and the antimicrobial peptide was designed to incorporate (Fig. 1B): (1) a proline residue at the C-terminal DAMP4, which has the lowest helix propensity allowing for breaking the preceding helix (Pace and Scholtz 1998); (2) a GGGGSGGGGS sequence providing a spatial separation of the pexiganan from the four-helix domain and flexibility (Chen et al. 2013); and (3) a thrombin recognition site (LVPRGS) sequence preceding pexiganan (Wu et al. 2008).

\section{Figure 1}

\section{Production of DAMP4-F-pexiganan}

We designed a fusion protein by fusing DAMP4 protein to a pexiganan peptide using a short linker (DPS) in our previous studies, denoted as “DAMP4-S-pexiganan” (Sun et al. 2018; Zhao et al. 2015). DAMP4-S-pexiganan has been demonstrated to be produced in E. coli cells by incubation with $1 \mathrm{mM} \mathrm{IPTG}$ at $25^{\circ} \mathrm{C}, 180 \mathrm{rpm}$ for $16 \mathrm{~h}$. The same condition was applied to produce DAMP4-F-pexiganan and the SDS-PAGE gel after cell lysis showed that DAMP4-F-pexiganan produced as evidenced from the protein band at around $15 \mathrm{kDa}$, which is close to the protein's theoretical MW (15,188.78 Da) and which is absent in non-induced cultures (data not shown). This result suggests that the recombinant $E$. coli can produce DAMP4-F-pexiganan at the same expression condition of the DAMP4-S-pexiganan.

In our previous work, we have developed a cost-effective purification procedure for DAMP4-fused proteins using a non-chromatography method (Sun et al. 2018; Wibowo et al. 2017). The method was adopted to purify DAMP4-F-pexiganan. Fig. S1 shows a flowchart of the purification process. Briefly, the DAMP4-Fpexiganan was first released into the bulk phase after breaking cell walls by sonication. Then, 0.5\% (w/v) PEI solution was added into the supernatant to remove E. coli DNA by forming PEI-DNA complexes. Subsequently, most soluble protein contaminants were denatured at a high temperature and salt stress condition, and subsequently 
removed as precipitate. The target protein DAMP4-F-pexiganan was further precipitated by a salting-out method using 1.8 $\mathrm{M} \mathrm{Na}_{2} \mathrm{SO}_{4}$ and recovered after a series of steps including rinsing, solubilizing and dialysis.

SDS-PAGE analysis was carried out on the supernatants taken after each purification step of DAMP4-Fpexiganan (Fig. 2A). As illustrated in Fig. 2A, the membrane fraction in cell lysate led to a vertical streak in the gel (Fig. 2A, lane 1) and many bands appeared more intense in the sample after DNA removal (Fig. 2A, lane 2). This is due to the high cell density of $E$. coli and the high amount of native proteins produced by $E$. coli. In addition, some unexpected bands between 10 and $30 \mathrm{kDa}$ were shown on the gel after the heating process (Fig. 2A, lane 3). Besides that, some protein bands (below $15 \mathrm{kDa}$ ) were found in the re-solubilizing sample after isolation of DAMP4-F-pexiganan (Fig. 2A, lane 6). After dialysis, only one significant band corresponding to the DAMP4-Fpexiganan appeared on the gel with a MW of approximately $15 \mathrm{kDa}$ (Fig. 2A, lane 7).

The purity of DAMP4-F-pexiganan after purification was determined using RP-HPLC (Fig. 2B). Fig. 2B shows a major peak of DAMP4-F-pexiganan at the retention time of $28.983 \mathrm{~min}$, and several small peaks were observed with retention times from 15 to $25 \mathrm{~min}$. Furthermore, the result of MS (Fig. S2) revealed the mass identity $(15,191.56 \mathrm{Da})$ of the DAMP4-F-pexiganan protein with the retention time of $28.983 \mathrm{~min}$, which matches the predicted mass of DAMP4-F-pexiganan (15,188 Da) and thus confirmed the presence of DAMP4-F-pexiganan at the peak of 28.223 min after purification.

\section{Figure 2}

\section{Impact of linker on the antimicrobial activity of the fusion protein}

As stated in the previous studies (Zhao et al. 2015), DAMP4-S-pexiganan can release pexiganan peptides under acidic conditions, and the antimicrobial activity of the released peptides has been confirmed, whereas the whole protein DAMP4-S-pexiganan has exhibited no activity against tested bacteria. In this study, the antimicrobial activity of DAMP4-S-pexiganan and DAMP4-F-pexiganan were compared to investigate whether the different linkers affect the antimicrobial performance of the fusion proteins. DAMP4-S-pexiganan and DAMP4-Fpexiganan were obtained from the same expression condition (incubation with $1 \mathrm{mM}$ IPTG at $25^{\circ} \mathrm{C}, 180 \mathrm{rpm}$ for $16 \mathrm{~h}$ ) and purification procedures (the non-chromatographic method) (Sun et al. 2018; Wibowo et al. 2017; Zhao et al. 2015). The antimicrobial performance of the fusions was evaluated by MIC tests (Fig. 3). All the wells containing DAMP4-S-pexiganan became turbid, showing that this preparation had no antimicrobial activity at concentrations ranging from 0.16 to $10 \mu \mathrm{M}$, as well as DAMP4 alone did not exhibit any antimicrobial activity at the same concentration range, which are consistent with the findings of previous studies (Zhao et al. 2015). In contrast, there was no bacterial growth in the wells with $10 \mu \mathrm{M}$ and $5 \mu \mathrm{M}$ DAMP4-F-pexiganan, suggesting the antimicrobial activity with a MIC of $5 \mu \mathrm{M}$, which was same as that of the synthetic pexiganan.

\section{Figure 3}

\section{Effect of expression conditions on the purification results}

Many factors (e.g., pH, temperature, medium, inducer, etc.) can influence the expression of recombinant proteins in E. coli, including cell mortality, protein-production yields, protein folding, protein solubility (Georgiou 1988). For example, high temperature or high inducer concentration can contribute to a rapid synthesis of a heterologous protein but that protein might fail to form its native conformation resulting in insoluble aggregates (Baneyx and Mujacic 2004). As aforementioned, it has been proven that the DAMP4-F-pexiganan can be produced under the low (L) temperature condition (incubation with $1 \mathrm{mM}$ IPTG at $25^{\circ} \mathrm{C}, 180 \mathrm{rpm}$ for $16 \mathrm{~h}$ ). To find an optimal expression condition for producing the DAMP4-F-pexiganan, a high (H) temperature condition (incubation with 
$1 \mathrm{mM}$ IPTG at $37^{\circ} \mathrm{C}, 180 \mathrm{rpm}$ for $4 \mathrm{~h}$ ) was performed to accelerate the rate of production of DAMP4-F-pexiganan. $\mathrm{L}$ and $\mathrm{H}$ conditions were investigated to study the effect on the structure, hence function, of the produced protein DAMP4-F-pexiganan. The DAMP4-F-pexiganan produced under these two conditions are referred as L-DAMP4F-pexiganan and H-DAMP4-F-pexiganan, respectively.

Similar to the production of L-DAMP4-F-pexiganan, a protein band at around $15 \mathrm{kDa}$ was observed on the SDS-PAGE gel of the cell lysate after production of H-DAMP4-F-pexiganan, while not found in the noninduced cultures (data not shown), indicating that the recombinant E. coli can produce DAMP4-F-pexiganan regardless of the expression condition at high or low temperatures.

Subsequently, the H-DAMP4-F-pexiganan was purified by the same procedures as for L-DAMP4-Fpexiganan and the purification results were analyzed by SDS-PAGE. Compared with L-DAMP4-F-pexiganan, the SDS-PAGE analysis of the purification of H-DAMP4-F-pexiganan shows some differences (Fig. 4A). There was no streak in the sample of the cell lysate (Fig. 4A, lane 1) and the protein contaminants detected in the sample after DNA removal were less significant (Fig. 4A, lane 2) due to the shorter expression time. Several unwanted bands were also observed in the sample after the heating process (Fig. 4A, lane 3) and the re-solubilizing sample (Fig. 4A, lane 6), whereas the bands were different from the result for the L-DAMP4-F-pexiganan (Fig. 2A, lane 3 and lane 6). Specifically, one strong band at approximately $10 \mathrm{kDa}$ was not observed and some bands ranging from 40 to $60 \mathrm{kDa}$ appeared on the gel (Fig. 4A, lane 3). After dialysis, only the band of H-DAMP4-F-pexiganan at around $15 \mathrm{kDa}$ was visible, which is consistent with the purification result of L-DAMP4-F-pexiganan (Fig. 2A, lane 7).

In contrast to purified L-DAMP4-F-pexiganan, the HPLC analysis of purified H-DAMP4-F-pexiganan after dialysis (Fig. 4B) only shows one single peak at 28.223 min corresponding to the H-DAMP4-F-pexiganan, suggesting that the non-chromatographic purification is able to achieve better purification of the target protein for the H-DAMP4-F-pexiganan with higher purity. Moreover, MS (Fig. S3) also showed a mass of 15,190.84 Da for H-DAMP4-F protein at the retention time of 28.223 min, which is also in good agreement with the theoretical mass, confirming the peak at the retention time of $28.223 \mathrm{~min}$ as the H-DAMP4-F-pexiganan.

\section{Figure 4}

\section{Effect of expression conditions on the antimicrobial activity of the fusion proteins}

Following the purification, MIC tests were performed to determine the antimicrobial activity of L-DAMP4-Fpexiganan and H-DAMP4-F-pexiganan. As shown in Fig 5A, L-DAMP4-F-pexiganan displayed the antimicrobial activity at the concentration higher than $5 \mu \mathrm{M}$ as same as the synthetic pexiganan, whereas the H-DAMP4-Fpexiganan did not inhibit the growth of bacteria at the same concentrations, suggesting the critical role of the effect of expression temperatures on the antimicrobial activity of the fusion proteins.

To further explore the reduced antimicrobial activity of H-DAMP4-F-pexiganan, the protein was cleaved by thrombin at the thrombin recognition site in the linker. The detailed method is described in the Supporting Information. Following thrombin digestion, the product was analyzed by SDS-PAGE and RP-HPLC. Although the released pexiganan cannot be clearly observed in the SDS-PAGE gel due to its small size (2,936.67 Da), the fusion proteins, L-DAMP4-F-pexiganan and H-DAMP4-F-pexiganan, were both reduced in size following digestion and both samples showed a band around $13 \mathrm{kDa}$, which is similar to the expected size of DAMP4 plus linker (12,309.73 Da) (Fig. S4). In addition, released pexiganan was detected by HPLC (Fig. S5) and MS confirmed that the molecular weights of GS-pexiganan-HH were 2,940.77 Da and 2,939.33 Da when released 
from L-DAMP4-F-pexiganan and H-DAMP4-F-pexiganan, respectively (Fig. S6). Furthermore, the DAMP4 fragments after digestion were found to be 12,308.64 $\mathrm{Da}$ and 12,306.40 Da. These results confirm the release of GS-pexiganan-HH by specific thrombin cleavage.

MIC tests showed that GS-pexiganan-HH from H-DAMP4-F-pexiganan did not have antimicrobial activity (Fig. 5B). In contrast, GS-pexiganan-HH from L-DAMP4-F-pexiganan possessed antimicrobial activity at the concentrations of $10 \mu \mathrm{M}$ and $5 \mu \mathrm{M}$. Mixing DAMP4 and synthetic pexiganan peptides showed the same MIC result as that of the pexiganan peptide alone, indicating that the DAMP4 protein did not influence the antimicrobial activity of the pexiganan peptide. This is in agreement with our previous study that showed no antimicrobial activity from DAMP4 alone (Zhao et al. 2015).

To further investigate the relationship between the structure and antimicrobial activity of DAMP4-Fpexiganan, a denaturation and refolding process was carried out on the H-DAMP4-F-pexiganan to promote refolding of the pexiganan domain into the active conformation (see the Supporting Information). Subsequent MIC tests showed that the refolded H-DAMP4-F-pexiganan possessed a similar MIC of $5 \mu \mathrm{M}$ as that of the L-DAMP4F-pexiganan and synthetic pexiganan (Fig. 5C (a)). This suggests that the antimicrobial activity of the H-DAMP4F-pexiganan was recovered after refolding. Furthermore, the GS-pexiganan-HH released from the refolded HDAMP4-F-pexiganan by thrombin digestion also showed antimicrobial activity with an identical MIC value (5 $\mu \mathrm{M}$ ) to the synthetic pexiganan (Fig. 5C (b)), demonstrating that the structure of pexiganan plays a critical role in its antimicrobial activity.

\section{Figure 5}

\section{Discussion}

In this work, we designed a novel fusion protein having excellent antimicrobial activity, DAMP4-F-pexiganan. This protein comprises a four-helix bundle DAMP4 domain linked to an antimicrobial peptide pexiganan via a long, flexible linker called "F" (PGGGGSGGGGSLVPRGS). Based on this rational design, it was hypothesized that the DAMP4-F-pexiganan would be easily produced and purified from E. coli due to the intrinsic stability of four-helix DAMP4 (Schaller et al. 2015), and that the linker F would allow the pexiganan domain to orient away from the rigid DAMP4 domain and retain its antimicrobial activity (Fig. 1A). This hypothesis implies that the antimicrobial function of the protein could be structure dependent, that is, dependent on how the pexiganan peptides are allowed to orientate and thus interact with bacteria. In addition, the peptide could be cleaved and released to have antimicrobial activity as well, if necessary or desired, especially in the event that thrombin would be available, for example, at a skin wound site (Fig. 1B) (Lipsky et al. 2008; Mangoni et al. 2016).

Due to the DAMP4 carrier, which is highly soluble and stable under extremely high temperature (up to $110^{\circ} \mathrm{C}$ ) and high salt conditions (Dwyer et al. 2014), DAMP4-F-pexiganan has the unique opportunity to be purified using a simple and cheap method based on heating and salting-out. As shown in the results of SDS-PAGE and HPLC analysis, we can observe that most contaminants have been removed after purification, but some contaminants remained after the whole process (Fig. 2A). One possible explanation is that some unwanted protein bands below $15 \mathrm{kDa}$ corresponded to degraded DAMP4-F-pexiganan following proteolysis in the host cells, and that the bands between 20 and $30 \mathrm{kDa}$ represented the aggregations of the fractions of DAMP4-F-pexiganan. These fractions have the ability to resist heating process because they could maintain their partial stability against the high salt stress and high temperature, and then salting out method resulted in co-precipitation of DAMP4-Fpexiganan and these protein fragments. Although dialysis is capable of precipitating some residual low molecular 
weight contaminants, the unexpected peaks were detected in the HPLC results between 15 to 25 min, which may represent proteolytic fragments of the DAMP4-F-pexiganan, suggesting that they were not completely removed (Fig. 2B).

Furthermore, in order to examine the role of linker length and composition in the antimicrobial activity of fusions, MIC test were performed to determine the antimicrobial activity of DAMP4-F-pexiganan that has the linker $\mathrm{F}$ of 17 residues including two flexible $\mathrm{G}_{4} \mathrm{~S}$ motifs, and DAMP4-S-pexiganan containing a short linker of 3 residues (DPS) from our previous study (Zhao et al. 2015). Only DAMP4-F-pexiganan can exhibit antimicrobial activity with a similar MIC of $5 \mu \mathrm{M}$ as that of the antimicrobial peptide (Fig. 3). This difference between DAMP4F-pexiganan and DAMP4-S-pexiganan indicates the antimicrobial activity of the pexiganan domain requires sufficient flexibility and separation from the DAMP4 domain. The limited flexibility of linker in DAMP4-Spexiganan negatively influenced pexiganan activity, resulting in a reduced ability of the peptide to attach to the membrane of target cells with the correct orientation for antimicrobial activity. By contrast, the long, flexible linker in DAMP4-F-pexiganan serves as a spacer to separate the pexiganan motif and DAMP4 carrier, and thus facilitates the exposing of pexiganan motif to target membranes for membrane disruption. Similar results have been reported by other groups investigating the effect of linker lengths on the activity of peptides immobilized to solid substrates (Han et al. 2014).

In this work, a crucial parameter in the production of DAMP4-F-pexiganan, the expression condition, was also investigated and discussed. As shown in the results, the $\mathrm{L}$ condition requires a long incubation time to produce sufficient amounts of L-DAMP4-F-pexiganan, which is time-consuming as well as increases the risk of proteolysis of fusions by host cells, thus resulting in that some protein fragments remained in the final product after purification. On the other hand, the H-DAMP4-F-pexiganan, which was produced under the $\mathrm{H}$ condition can be purified through the same process as the L-DAMP4-F-pexiganan. However, compared with the purification results of L-DAMP4-F-pexiganan, SDS-PAGE gel showed different protein contaminants bands in the purification process of H-DAMP4-F-pexiganan and the HPLC result suggested a higher purity of H-DAMP4-F-pexiganan after purification (Fig. 4). This difference was likely caused by the different extent of aggregation and proteolysis under different temperatures and expression time.

Overall, both the L-DAMP4-F-pexiganan and H-DAMP4-F-pexiganan can be purified using the nonchromatographic purification process. However, proteolytic products and their aggregations were detected at intermediate stages of the purification, and the yields of the purified L-DAMP4-F-pexiganan and H-DAMP4-Fpexiganan were not as high as previously reported for fusions with DAMP4 carrier, such as DAMP4-S-pexiganan (24 mg per $800 \mathrm{~mL}$ cell culture (OD $\left.{ }_{600} \sim 2\right)$ ) and D4S2 protein (28.8 mg per $800 \mathrm{~mL}$ cell culture $\left(\mathrm{OD}_{600} \sim 2\right)$ ) (Sun et al. 2018; Wibowo et al. 2017). One possible reason is that the long linker increases the flexibility of pexiganan, increasing susceptibility to proteolysis by the host cell and resulting in a lower concentration of intact fusion protein in the starting material.

Moreover, the expression condition also plays a critical role in the antimicrobial activity of the fusion protein. The H-DAMP4-F-pexiganan cannot exhibit any antimicrobial activity in the MIC test. This is opposite to the antimicrobial result of L-DAMP4-F-pexiganan, which has a MIC value of $5 \mu \mathrm{M}$ (Fig. 5A). This difference might be due to more precise folding of DAMP4-F-pexiganan during production at the lower temperature. Previous work has shown similar results using green fluorescent protein, which possessed a better solubility and conformational quality when produced at a low temperature in E. coli (Martínez-Alonso et al. 2008). 
In addition, GS-pexiganan-HH were released from DAMP4-F-pexiganan and investigated separately. Both LDAMP4-F-pexiganan and H-DAMP4-F-pexiganan produce the GS-pexiganan-HH with correct sequence (Fig. S4-S6), but only the GS-pexiganan-HH from L-DAMP4-F-pexiganan displayed the antimicrobial activity (Fig. 5B). Therefore, we speculate that the structure of the GS-pexiganan-HH produced at high temperature is misfolded and only appropriate expression conditions (longer expression time and low temperature) allow correct structure and an active conformation of the peptide. What is more, after the denaturation and refolding process, the $\mathrm{H}$ DAMP4-F-pexiganan and its released GS-pexiganan-HH successfully showed same MIC values as produced in the L expression conditions (Fig. 5C). Considering all the data presented, we can conclude that the conformation of pexiganan could differ significantly under different expression conditions, reflected by the different antimicrobial performance of the recombinant DAMP4-F-pexiganan proteins.

In summary, a novel fusion protein DAMP4-F-pexiganan was designed in this work. It can be purified based on a non-chromatography method as well as display antimicrobial activity without the release of AMPs. Interestingly, the antimicrobial activity of the DAMP4-F-pexiganan depends on both linker characteristics and expression conditions. A long, flexible linker allow pexiganan to adopt an active conformation in fusion with DAMP4 and attach to pathogen membranes. Also, the fusion protein requires a low temperature condition to be produced with the pexiganan in an active conformation, although the necessarily longer induction time can yield more protein with fewer protein contaminants in a short time period, but the pexiganan domain was not active. This work will open up a new way in the development of antimicrobial agents, and the studies on expression conditions and linker will serve as a guide to help the rational design of fusion proteins and their purification processes. 


\section{Acknowledgments}

This research was supported by the Australian Research Council (ARC) under Discovery Project (DP150100798).

C.-X Zhao acknowledges financial support from the award of the Australian Research Council (ARC) Future Fellowship (FT140100726). We acknowledge the facilities of Australian Genome Research Facility, The University of Queensland for gene sequencing. This work was performed in part at the Queensland node of the Australian National Fabrication Facility (ANFF-Q), a company established under the National Collaborative Research Infrastructure Strategy to provide nano and micro-fabrication facilities for Australia’s researchers.

\section{Compliance with ethical standards}

\section{Conflict of interests}

The authors declare no financial or commercial conflict of interest.

\section{Ethical statement}

This article does not contain any studies involving human participants or experimental animals. 


\section{References}

Ageitos J, Sánchez-Pérez A, Calo-Mata P, Villa T (2017) Antimicrobial peptides (AMPs): ancient compounds that represent novel weapons in the fight against bacteria. Biochem Pharmacol 133:117-138 doi:10.1016/j.bcp.2016.09.018

Baneyx F, Mujacic M (2004) Recombinant protein folding and misfolding in Escherichia coli. Nat Biotechnol 22(11):1399 doi:10.1038/nbt1029

Brogden KA (2005) Antimicrobial peptides: pore formers or metabolic inhibitors in bacteria? Nature reviews microbiology 3(3):238 doi:doi:10.1038/nrmicro1098

Capparelli R, Ventimiglia I, Palumbo D, Nicodemo D, Salvatore P, Amoroso MG, Iannaccone M (2007) Expression of recombinant puroindolines for the treatment of staphylococcal skin infections (acne vulgaris). J Biotechnol 128(3):606-614 doi:10.1016/j.jbiotec.2006.11.004

Chen X, Zaro JL, Shen W-C (2013) Fusion protein linkers: property, design and functionality. Adv Drug Del Rev 65(10):1357-1369 doi:10.1016/j.addr.2012.09.039

Davies J, Davies D (2010) Origins and evolution of antibiotic resistance. Microbiol Mol Biol Rev 74(3):417-433 doi:10.1128/mmbr.00016-10

Dwyer MD, Brech M, Yu L, Middelberg AP (2014) Intensified expression and purification of a recombinant biosurfactant protein. Chem Eng Sci 105:12-21 doi:10.1016/j.ces.2013.10.024

Fox JL (2013) Antimicrobial peptides stage a comeback. Nat Biotechnol 31(5):379-382 doi:10.1038/nbt.2572

Georgiou G (1988) Optimizing the production of recombinant proteins in microorganisms. AICHE J 34(8):12331248 doi:10.1002/aic.690340802

Gibbs GM, Davidson BE, Hillier AJ (2004) Novel expression system for large-scale production and purification of recombinant class IIa bacteriocins and its application to piscicolin 126. Appl Environ Microbiol 70(6):3292-3297 doi:10.1128/aem.70.6.3292-3297.2004

Gottler LM, Ramamoorthy A (2009) Structure, membrane orientation, mechanism, and function of pexiganan-a highly potent antimicrobial peptide designed from magainin. Biochimica et Biophysica Acta (BBA)Biomembranes 1788(8):1680-1686 doi:10.1016/j.bbamem.2008.10.009

Guaní-Guerra E, Santos-Mendoza T, Lugo-Reyes SO, Terán LM (2010) Antimicrobial peptides: general overview and clinical implications in human health and disease. Clin Immunol 135(1):1-11 doi:10.1016/j.clim.2009.12.004

Han X, Liu Y, Wu F-G, Jansensky J, Kim T, Wang Z, Brooks III CL, Wu J, Xi C, Mello CM (2014) Different interfacial behaviors of peptides chemically immobilized on surfaces with different linker lengths and via different termini. The Journal of Physical Chemistry B 118(11):2904-2912 doi:10.1021/jp4122003

Lee J, Kim J, Hwang S, Lee W, Yoon H, Lee H, Hong S (2000) High-level expression of antimicrobial peptide mediated by a fusion partner reinforcing formation of inclusion bodies. Biochem Biophys Res Commun 277(3):575-580 doi:10.1006/bbrc.2000.3712

Li JF, Zhang J, Zhang Z, Kang CT, Zhang SQ (2011) SUMO mediating fusion expression of antimicrobial peptide CM4 from two joined genes in Escherichia coli. Curr Microbiol 62(1):296-300 doi:10.1007/s00284-0109705-3

Li Y (2009) Carrier proteins for fusion expression of antimicrobial peptides in Escherichia coli. Biotechnol Appl Biochem 54(1):1-9 doi:10.1042/ba20090087

Li Y (2011) Recombinant production of antimicrobial peptides in Escherichia coli: a review. Protein Expression Purif 80(2):260-267 doi:10.1016/j.pep.2011.08.001

Lipsky BA, Holroyd KJ, Zasloff M (2008) Topical versus systemic antimicrobial therapy for treating mildly infected diabetic foot ulcers: a randomized, controlled, double-blinded, multicenter trial of pexiganan cream. Clin Infect Dis 47(12):1537-1545 doi:10.1086/593185

Majerle A, Kidrič J, Jerala R (2000) Production of stable isotope enriched antimicrobial peptides in Escherichia coli: an application to the production of a $15 \mathrm{~N}$-enriched fragment of lactoferrin. J Biomol NMR 18(2):145-151 doi:doi:10.1023/a:1008362401928

Mangoni ML, McDermott AM, Zasloff M (2016) Antimicrobial peptides and wound healing: biological and therapeutic considerations. Exp Dermatol 25(3):167-173 doi:10.1111/exd.12929

Martínez-Alonso M, García-Fruitós E, Villaverde A (2008) Yield, solubility and conformational quality of soluble proteins are not simultaneously favored in recombinant Escherichia coli. Biotechnol Bioeng 101(6):13531358 doi:10.1002/bit.21996

Middelberg AP, Dimitrijev-Dwyer M (2011) A designed biosurfactant protein for switchable foam control. Chemphyschem 12(8):1426-1429 doi:10.1002/cphc.201100082

Pace CN, Scholtz JM (1998) A helix propensity scale based on experimental studies of peptides and proteins. Biophys J 75(1):422-427 doi:10.1016/s0006-3495(98)77529-0

Parachin NS, Mulder KC, Viana AAB, Dias SC, Franco OL (2012) Expression systems for heterologous production of antimicrobial peptides. Peptides 38(2):446-456 doi:10.1016/j.peptides.2012.09.020 
Pasupuleti M, Schmidtchen A, Malmsten M (2012) Antimicrobial peptides: key components of the innate immune system. Crit Rev Biotechnol 32(2):143-171 doi:10.3109/07388551.2011.594423

Schaller A, Connors NK, Oelmeier SA, Hubbuch J, Middelberg AP (2015) Predicting recombinant protein expression experiments using molecular dynamics simulation. Chem Eng Sci 121:340-350 doi:10.1016/j.ces.2014.09.044

Sun B, Wibowo D, Middelberg AP, Zhao C-X (2018) Cost-effective downstream processing of recombinantly produced pexiganan peptide and its antimicrobial activity. AMB Express 8(1):6 doi:10.1186/s13568-0180541-3

Toennies G, Homiller RP (1942) The oxidation of amino acids by hydrogen peroxide in formic acid. J Am Chem Soc 64(12):3054-3056 doi:doi:10.1021/ja01264a518

Vallejo LF, Rinas U (2004) Strategies for the recovery of active proteins through refolding of bacterial inclusion body proteins. Microbial cell factories 3(1):11 doi:10.1186/1475-2859-3-11

Wibowo D, Yang G-Z, Middelberg AP, Zhao CX (2017) Non-chromatographic bioprocess engineering of a recombinant mineralizing protein for the synthesis of silica nanocapsules. Biotechnol Bioeng 114(2):335343 doi:10.1002/bit.26079

Wiegand I, Hilpert K, Hancock RE (2008) Agar and broth dilution methods to determine the minimal inhibitory concentration (MIC) of antimicrobial substances. Nat Protoc 3(2):163 doi:10.1038/nprot.2007.521

Wu G-Q, Li L-X, Ding J-X, Wen L-Z, Shen Z-L (2008) High-level expression and novel purification strategy of recombinant thanatin analog in Escherichia coli. Curr Microbiol 57(2):95 doi:10.1007/s00284-008-9106$\mathrm{z}$

Zhao CX, Dwyer MD, Yu AL, Wu Y, Fang S, Middelberg AP (2015) A simple and low-cost platform technology for producing pexiganan antimicrobial peptide in E. coli. Biotechnol Bioeng 112(5):957-964 doi:doi:10.1002/bit.25505

Zhou Q-F, Luo X-G, Ye L, Xi T (2007) High-level production of a novel antimicrobial peptide perinerin in Escherichia coli by fusion expression. Curr Microbiol 54(5):366-370 doi:10.1007/s00284-006-0466-y 


\section{Figure legends}

Figure 1. A) Cartoon of DAMP4-F-pexiganan - generated using VMD software; B) Sequence design of DAMP4F-pexiganan.

Figure 2. A) SDS-PAGE analysis of the purification process of DAMP4-F-pexiganan (L-DAMP4-F-pexiganan): The purification of L-DAMP4-F-pexiganan. Lane 1: after cell lysis; lane 2: after DNA removal; lane 3: after heating; lane 4: after isolation of DAMP4-F-pexiganan; lane 5: after rinsing; lane 6: after solubilizing; lane 7: after buffer exchange; marker, Precision Plus Protein ${ }^{\mathrm{TM}}$ Dual Xtra Prestained Protein Standards. All the samples were obtained from the supernatant; B) RP-HPLC profiles for characterization of the purified DAMP4-Fpexiganan (L-DAMP4-F-pexiganan).

Figure 3. Results of MIC assay of DAMP4-F-pexiganan (L-DAMP4-F-pexiganan) and DAMP4-S-pexiganan against E. coli ATCC ${ }^{\circledR} 25922^{\mathrm{TM}}$. GC, growth control (broth with bacterial inoculum, no proteins/peptides); SC, sterility control (broth only).

Figure 4. A) SDS-PAGE analysis of the purification process of the purification of H-DAMP4-F-pexiganan. Lane 1: after cell lysis; lane 2: after DNA removal; lane 3: after heating; lane 4: after rinsing; lane 5: after isolation of DAMP4-F-pexiganan; lane 6: after solubilizing; lane 7: after buffer exchange; marker, Novex BenchMark Protein Ladder. All the samples were obtained from the supernatant; B) RP-HPLC profiles for characterization of the purified DAMP4-F-pexiganan (H-DAMP4-F-pexiganan).

Figure 5. A) Results of MIC assay of L-DAMP4-F-pexiganan and H-DAMP4-F-pexiganan against E. coli ATCC ${ }^{\circledR}$ $25922^{\mathrm{TM}}$; B) Results of MIC assay of GS-pexiganan-HH released from L-DAMP4-F-pexiganan and H-DAMP4F-pexiganan against E. coli ATCC ${ }^{\circledR} 25922^{\mathrm{TM}}$. In the fifth row, Synthetic pexiganan peptide and DAMP4 protein was mixed with a molar ratio 1 to 1 ; $\mathbf{C}$ ) Results of MIC assay of (a) the refolded H-DAMP4-F-pexiganan and (b) the GS-pexiganan-HH released from refolded H-DAMP4-F-pexiganan. GC, growth control (broth with bacterial inoculum, no proteins/peptides); SC, sterility control (broth only). 


\section{Figure 1}

A

\section{B}

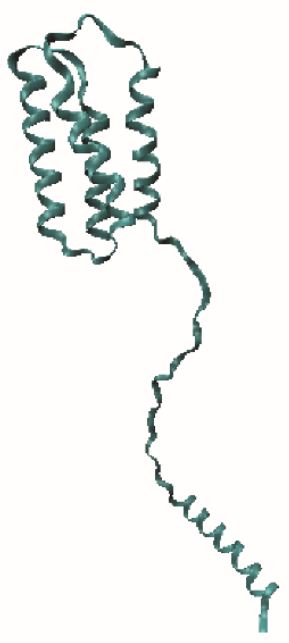

MDPSMKQLADSLHQLARQVSRLEHAD

PSMKQLADSLHQLARQVSRLEHAD

PSMKQLADSLHQLARQVSRLEHAD

PSMKQLADSLHQLARQVSRLEHAD

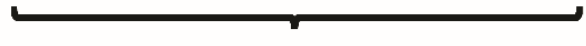

DAMP4 carrier

PGGGGSGGGGSLVPRGS GIGKFLKKAKKFGKAFVKILKKHH

Linker

Pexiganan 
Figure 2

A

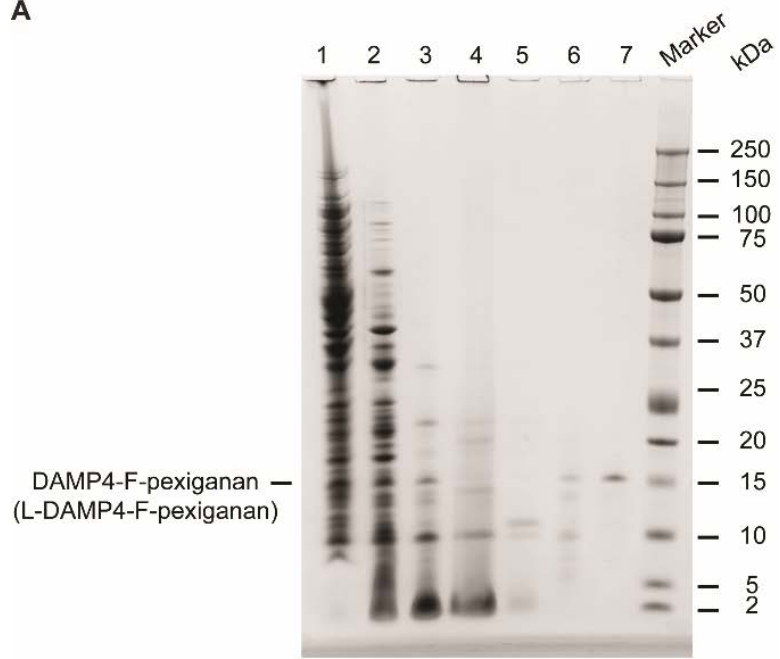

B

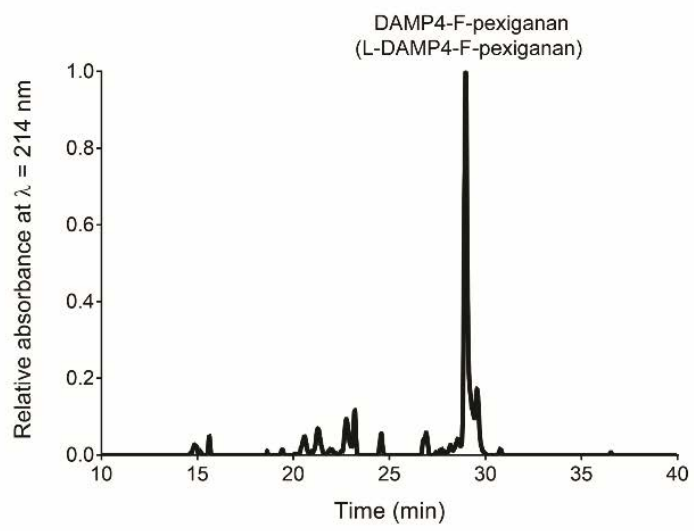




\section{Figure 3}

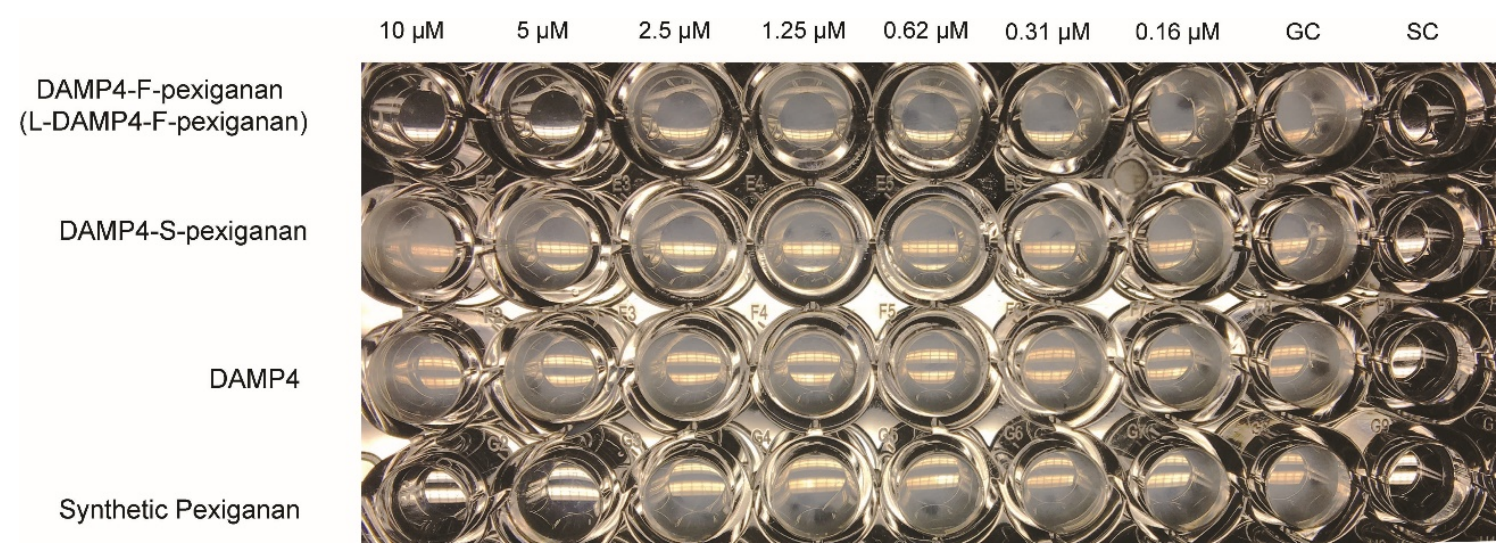


Figure 4

A

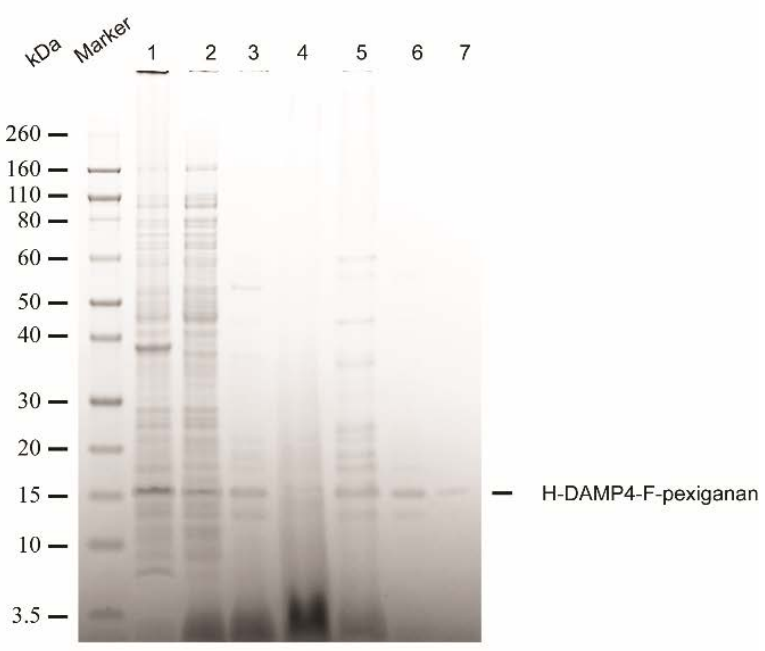

B

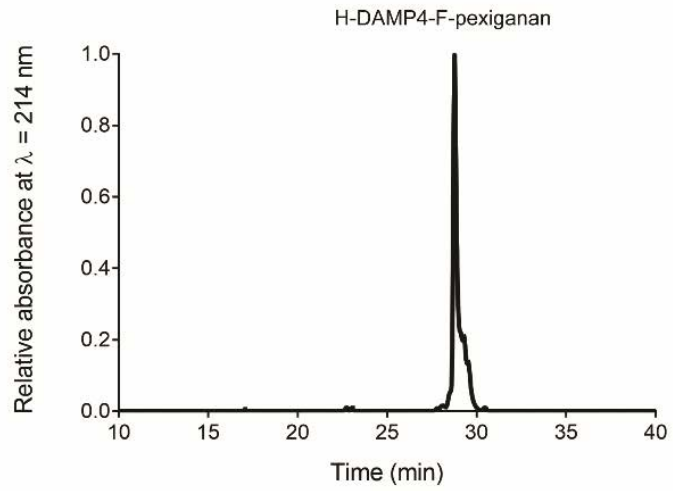




\section{Figure 5}

A

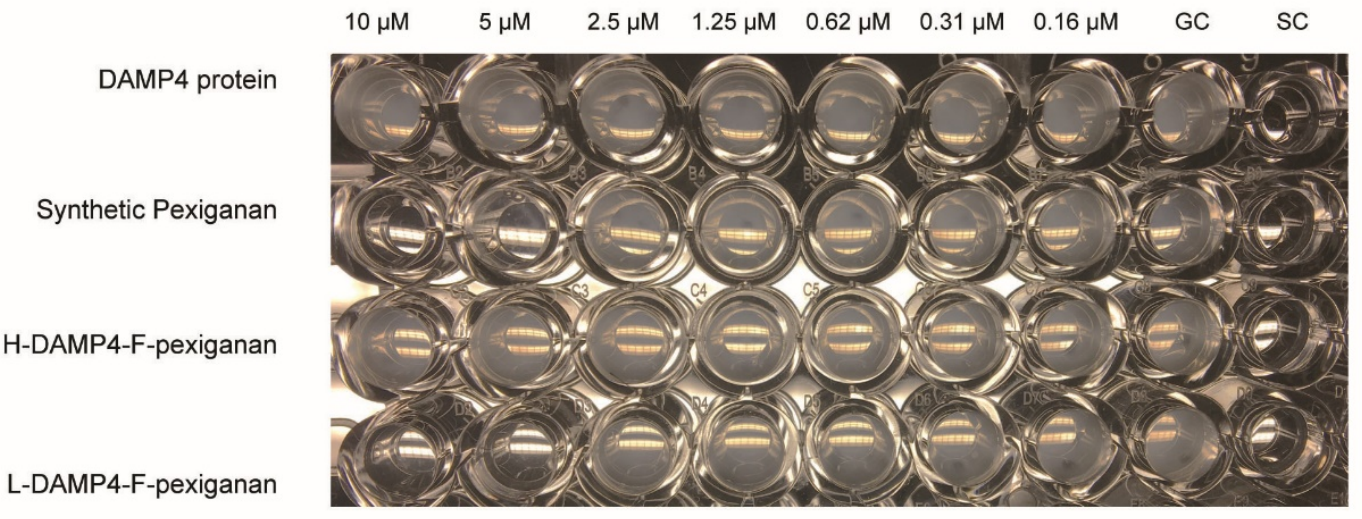

B

DAMP4 protein

GS-pexiganan-HH released from L-DAMP4-F-pexiganan

GS-pexiganan-HH released from H-DAMP4-F-pexiganan

Synthetic Pexiganan

Synthetic Pexiganan mixed with DAMP4 protein

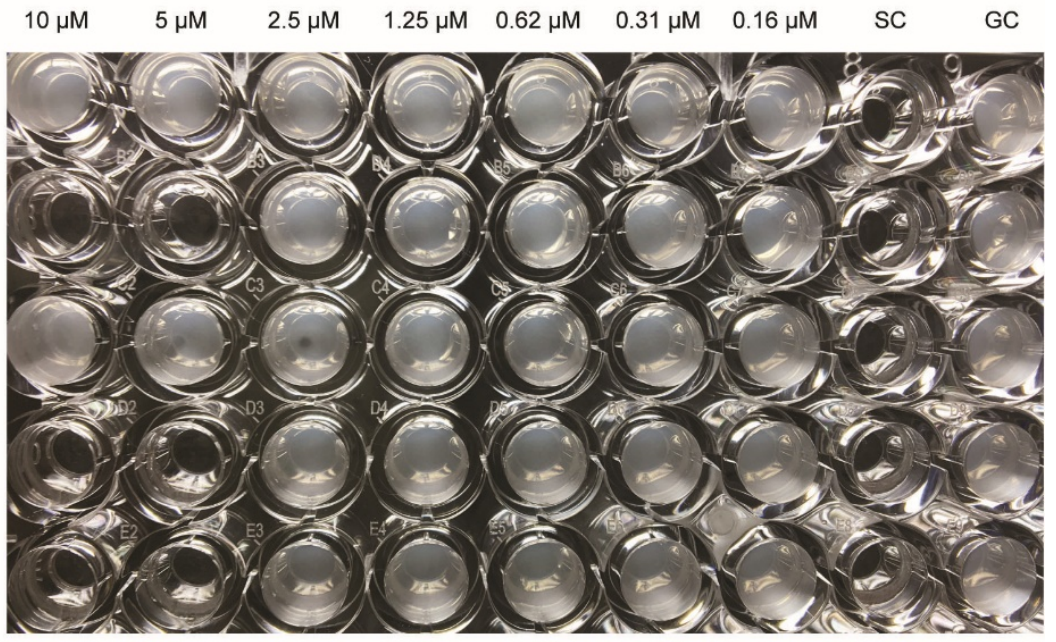

C

(a)

Refolded H-DAMP4-F-pexiganan H-DAMP4-F-pexiganan

$5 \mu \mathrm{M} \quad 2.5 \mu \mathrm{M} \quad 1.25 \mu \mathrm{M} \quad 0.62 \mu \mathrm{M} \quad 0.31 \mu \mathrm{M} \quad$ GC $\quad$ SC

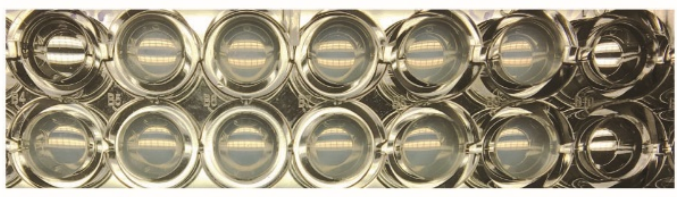

(b)

GS-Pexiganan-HH from refolded H-DAMP4-F-pexiganan

Synthetic Pexiganan

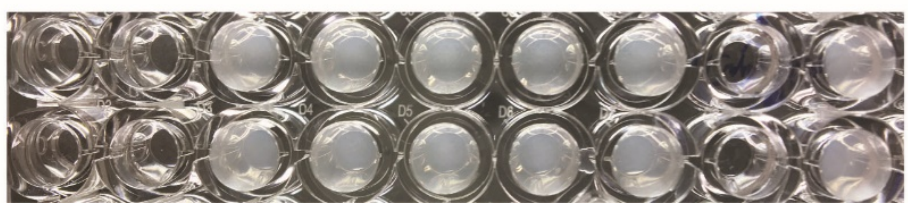




\section{Supporting information}

\section{Design and production of a novel antimicrobial fusion protein in}

Escherichia coli

Baode Sun, ${ }^{1}$ David Wibowo, ${ }^{1,2^{*}}$ Frank Sainsbury, ${ }^{1}$ Chun-Xia Zhao ${ }^{1 *}$

${ }^{1}$ Australian Institute for Bioengineering and Nanotechnology, The University of Queensland, St Lucia QLD 4072, Australia.

${ }^{2}$ Griffith Institute for Drug Discovery, Griffith University, Nathan QLD 4111, Australia.

*Correspondence

David Wibowo. Email: d.wibowo@griffith.edu.au. ORCID ID: 0000-0001-6919-3355

Chun-Xia Zhao. Email: z.chunxia@uq.edu.au. ORCID ID: 0000-0002-3365-3759

\section{Keywords}

antimicrobial agents; fusion proteins; minimum inhibitory concentration; protein purification; recombinant E. coli 


\section{Release of GS-pexiganan-HH from DAMP4-F-pexiganan by thrombin digestion}

The release of the pexiganan peptide from the DAMP4-F-pexiganan was carried out with thrombin. The purified DAMP4-F-pexiganan in HEPES buffer (10 mM, pH 7.2) was incubated with $20 \mathrm{U} / \mathrm{ml}$ thrombin at room temperature for $16 \mathrm{~h}$. The digestion results were analyzed using SDS-PAGE gel (Fig. S4), RP-HPLC (Fig. S5). The masses of the product after digestion were further identified by mass spectrometry (Fig. S6).

\section{Denaturation and refolding of GS-pexiganan-HH from H-DAMP4-F-pexiganan}

Chemical denaturation of DAMP4-F-pexiganan was carried out using the chaotropic agent. 4 M guanidine hydrochloride $(\mathrm{GdmCl})$ was added in the purified H-DAMP4-F-pexiganan in HEPES buffer (10 mM pH 7.2) and followed by stirring at room temperature for 2 h. And then, the one-step dialysis was used to refold the DAMP4-F-pexiganan. The denatured H-DAMP4F-pexiganan was refolded by dialysis against HEPES buffer (10 mM, pH 7.2) using SnakeSkin ${ }^{\circledR}$ pleated dialysis membrane (10,000 molecular weight cut off) (Thermo Fisher Scientific, North Ryde, Australia) at $4^{\circ} \mathrm{C}$, overnight.

\section{Table}

Table S1. Sequence of primers used in PCR.

\begin{tabular}{|c|c|}
\hline Primers & Primers sequence ( from 5' end to 3' end ) \\
\hline Primer-F & ACTTTAAGAAGGAGATATACATATG \\
\hline Primer-R & TGTACAGAATTCGGATCC \\
\hline
\end{tabular}




\section{Figures}

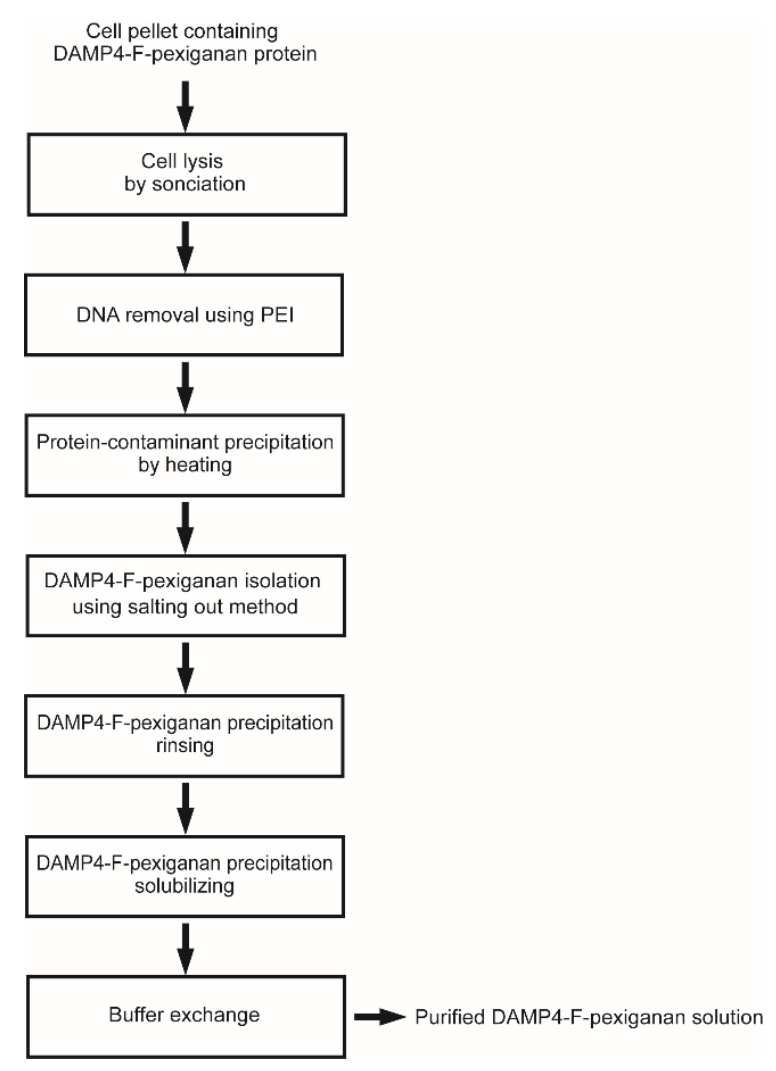

Figure S1. Block flow diagram of the purification process for the DAMP4-F-pexiganan. Step 1: cell lysis in lysis buffer by sonication; step 2: removal of DNA using $0.5 \%$ (w/v) PEI; step 3: precipitation of protein-contaminants using $1 \mathrm{M} \mathrm{Na}_{2} \mathrm{SO}_{4}\left(90^{\circ} \mathrm{C}, 30 \mathrm{~min}\right)$; step 4: precipitation of DAMP4-F-pexiganan using $1.8 \mathrm{M} \mathrm{Na}_{2} \mathrm{SO}_{4}\left(30^{\circ} \mathrm{C}, 60 \mathrm{~min}\right)$; step 5: rinsing of DAMP4-Fpexiganan precipitate using rinsing buffer; step 6: solubilizing of DAMP4-F-pexiganan precipitate in solubilizing buffer; step 7: buffer exchange using dialysis against HEPES buffer (10 mM pH 7.2). Lysis buffer: $25 \mathrm{mM}$ Tris-HCl, $1 \mathrm{M} \mathrm{NaCl,} \mathrm{pH} \mathrm{8.} \mathrm{Solubilizing} \mathrm{buffer:} 25 \mathrm{mM}$ Tris-HCl, $1 \mathrm{M} \mathrm{NaCl}$, pH 8. Rinsing buffer: 25mM Tris-HCl, $1 \mathrm{M} \mathrm{NaCl,} \mathrm{2.4M} \mathrm{Na} 2 \mathrm{SO}_{4}, \mathrm{pH} 8$. 


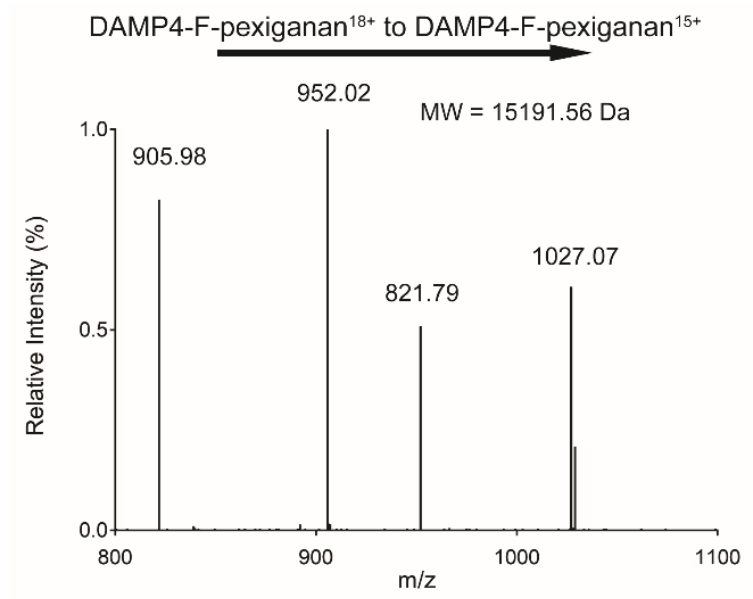

Figure S2. Mass spectrometry of the purified DAMP4-F-pexiganan (L-DAMP4-F-pexiganan)

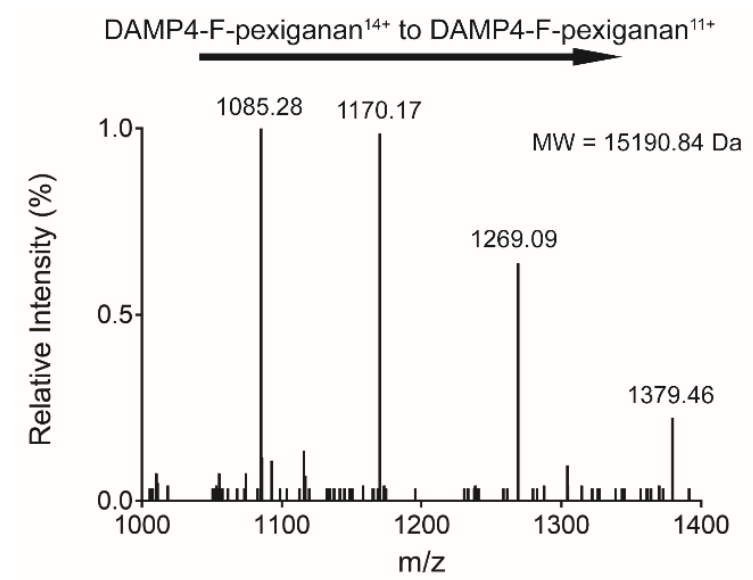

Figure S3. Mass spectrometry of the purified DAMP4-F-pexiganan (H-DAMP4-F-pexiganan) 


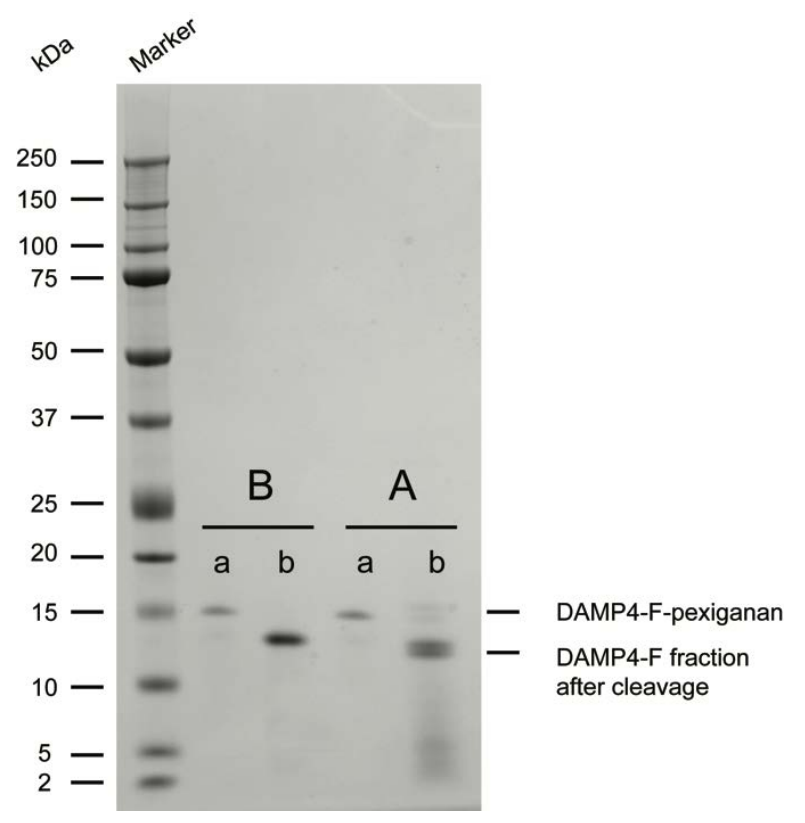

Figure S4. Results of SDS-PAGE analysis of thrombin digestion of (A) L-DAMP4-Fpexiganan and (B) H-DAMP4-F-pexiganan. Lane a: DAMP4-F-pexiganan before digestion; lane b: DAMP4-F-pexiganan after digestion; marker, Precision Plus Protein ${ }^{\mathrm{TM}}$ Dual Xtra Prestained Protein Standards.

(a)

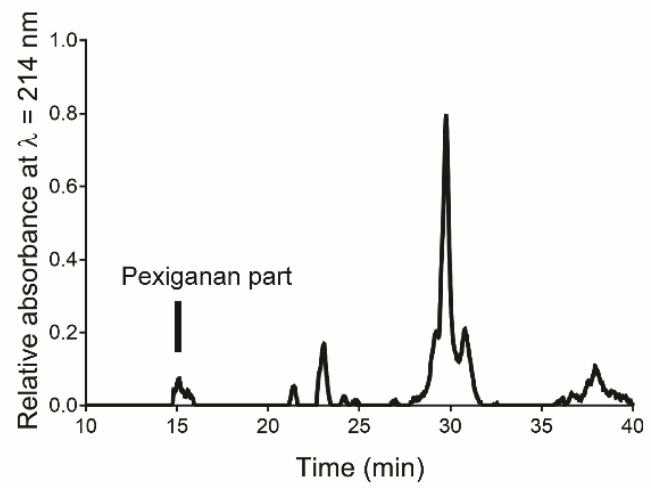

(b)

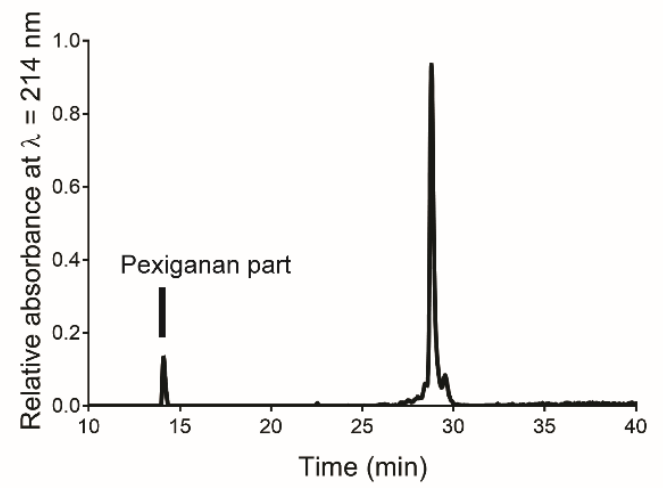

Figure S5. Characterization of the digestion result of (a) L-DAMP4-F-pexiganan and (b) HDAMP4-F-pexiganan using PR-HPLC.

Note: Based on the previous studies on the cleavage of the fusion protein comprising a DAMP4 protein and a pexiganan peptide, the peaks at retention time around 15 min represent the released pexiganan part after cleavage (Zhao et al. 2015). 
(a) PS-pexiganan-HH peptides released from L-DAMP4-F-pexiganan

PS-pexiganan- $\mathrm{HH}^{9+}$ to PS-pexiganan- $\mathrm{HH}^{7+}$

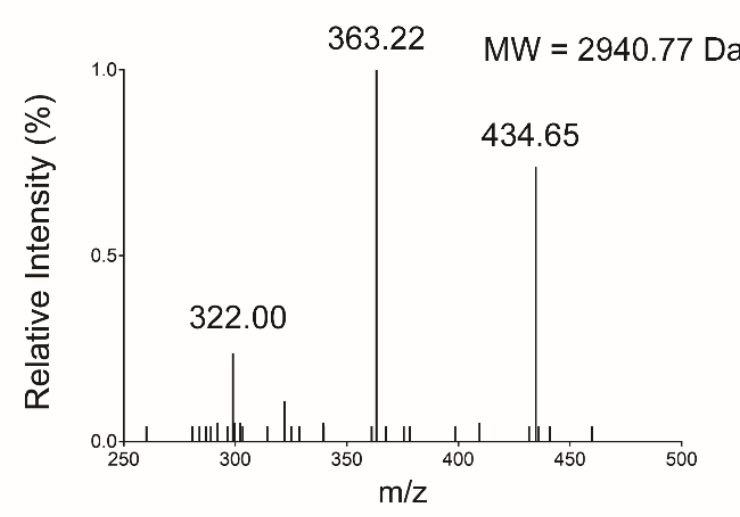

(c) DAMP4 fraction of L-DAMP4-F-pexiganan DAMP4 fraction ${ }^{16+}$ to DAMP4 fraction ${ }^{13+}$

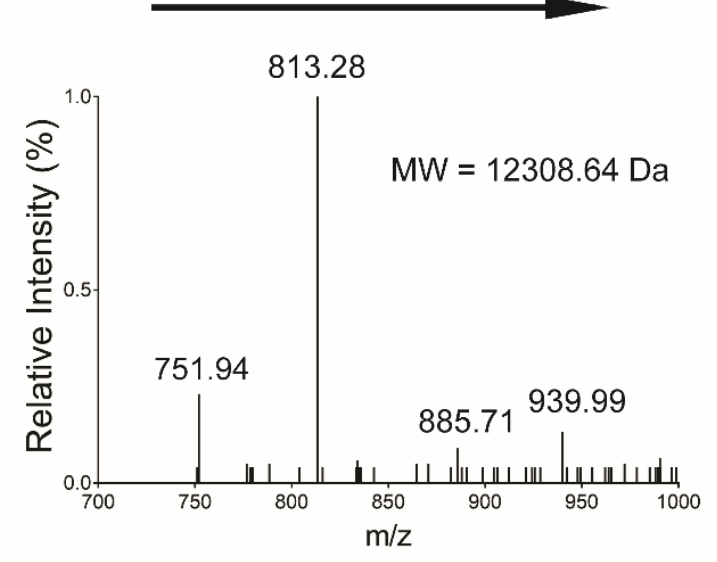

PS-pexiganan-HH peptides
(b) released from H-DAMP4-F-pexiganan

PS-pexiganan- $\mathrm{HH}^{13+}$ to PS-pexiganan- $\mathrm{HH}^{11+}$

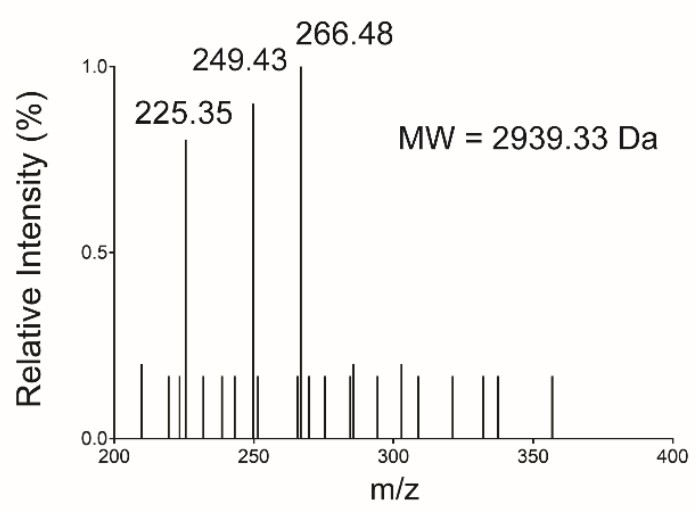

(d) DAMP4 fraction of H-DAMP4-F-pexiganan

DAMP4 fraction ${ }^{12+}$ to DAMP4 fraction ${ }^{9+}$

1394.76

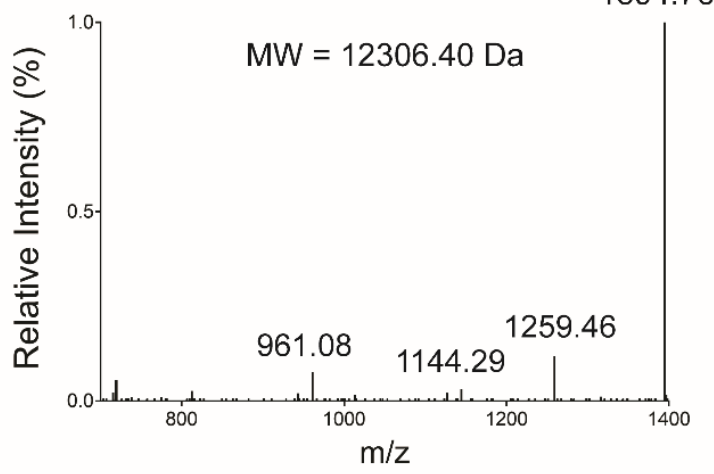

Figure S6. Mass spectrometry of (a) GS-pexiganan-HH peptides released from L-DAMP4-Fpexiganan; (b) GS-pexiganan-HH peptides released from H-DAMP4-F-pexiganan; (c) DAMP4 fraction after thrombin digestion of L-DAMP4-F-pexiganan; (d) DAMP4 fraction after thrombin digestion of H-DAMP4-F-pexiganan.

\section{Supporting reference}

Zhao CX, Dwyer MD, Yu AL, Wu Y, Fang S, Middelberg APJ (2015) A simple and low-cost platform technology for producing pexiganan antimicrobial peptide in E. coli. Biotechnol Bioeng 112(5): 957-964 